Document downloaded from:

http://hdl.handle.net/10251/126487

This paper must be cited as:

Bonet Solves, JA. (2018). THE FRECHET SCHWARTZ ALGEBRA OF UNIFORMLY CONVERGENT DIRICHLET SERIES. Proceedings of the Edinburgh Mathematical Society. 61(4):933-942. https://doi.org/10.1017/S0013091517000438

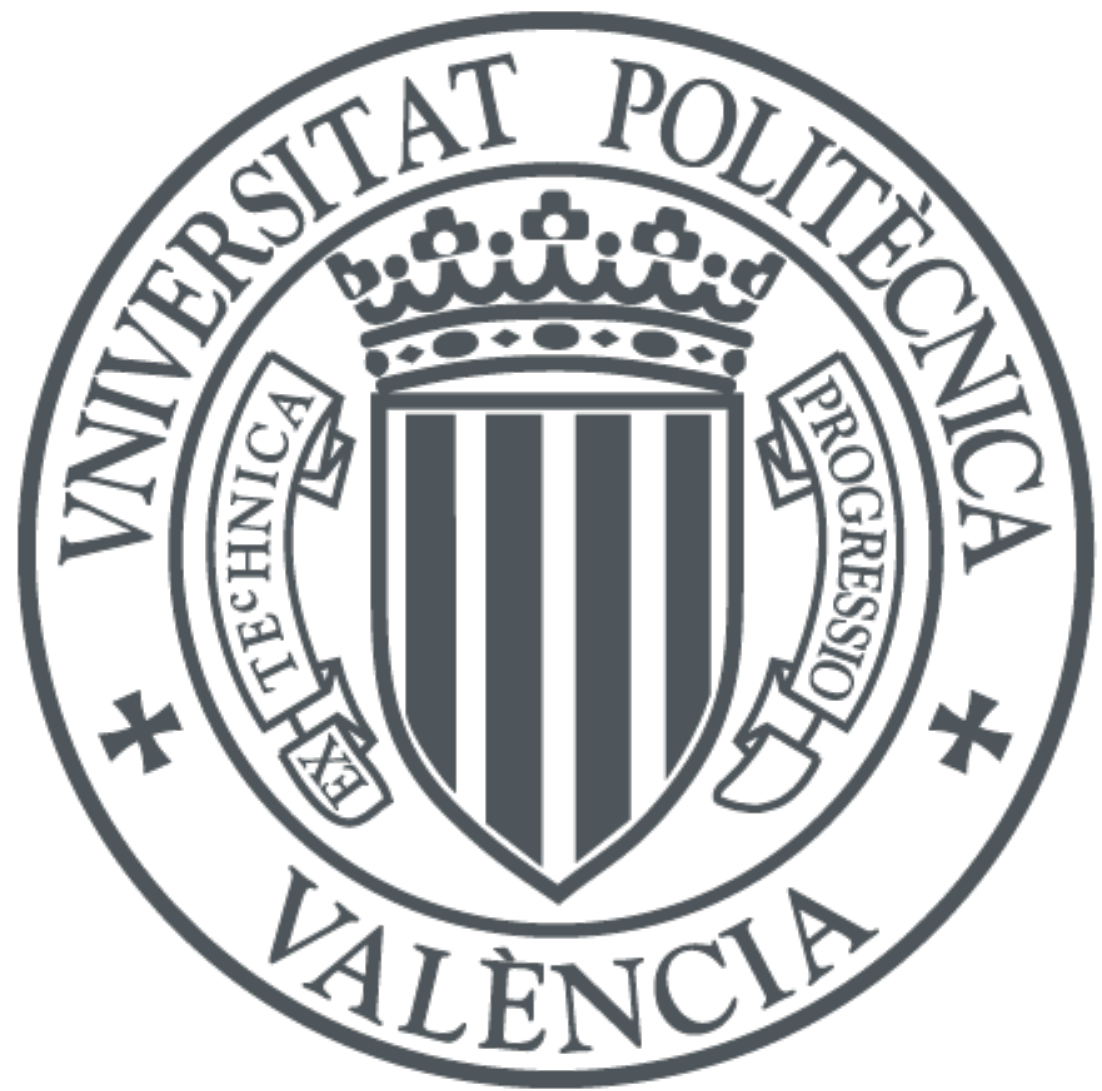

The final publication is available at

http://doi.org/10.1017/S0013091517000438

Copyright Cambridge University Press

Additional Information 


\title{
The Fréchet Schwartz algebra of uniformly convergent Dirichlet series
}

\author{
José Bonet
}

\begin{abstract}
The algebra of all Dirichlet series that are uniformly convergent in the half-plane of complex numbers with positive real part is investigated. When it is endowed with its natural locally convex topology it is a non-nuclear Fréchet Schwartz space with basis. Moreover, it is a locally multiplicative algebra but not a $Q$-algebra. Composition operators on this space are also studied.
\end{abstract}

\section{Introduction and preliminaries}

We study the Fréchet space $\mathcal{H}_{+}^{\infty}$ of uniformly convergent Dirichlet series in the half-plane $\mathbb{C}_{0}:=\{s \in \mathbb{C} \mid \operatorname{Re} s>0\}$. When endowed with its natural topology it is Schwartz, not nuclear, has a basis and contains isomorphically the space $H\left(\mathbb{D}^{m}\right)$ of analytic functions on the open unit polydisc $\mathbb{D}^{m}$ for each $m \in \mathbb{N}$; see Theorem 2.2. Moreover, this space is a multiplicatively convex Fréchet algebra for the pointwise product, that is not a $Q$-algebra, i.e. the set of invertible elements is not open, see Theorem 2.6. Composition operators on this algebra are studied in Section 3. The motivation for the research in this note comes from two results about bounded Dirichlet series. The first one is a classical result of Bohr, that is of central importance in the study of Dirichlet series. It asserts that if $f \in \mathcal{H}^{\infty}$, i.e. if $f$ is a bounded analytic function on the half complex plane $\mathbb{C}_{0}$ and it can be represented as a convergent Dirichlet series $f(s)=\sum_{n} a_{n} n^{-s}$ for Re $s$ large enough, then the Dirichlet series converges uniformly in each half-plane $\mathbb{C}_{\varepsilon}:=\{s \in \mathbb{C} \mid \operatorname{Re} s>\varepsilon\}, \varepsilon>0$; see Theorem 6.2.3 in [31] and [10]. This means that the abscissa of uniform convergence $\sigma_{u}(f)$ of $f$ satisfies $\sigma_{u}(f) \leq 0$. It also implies that the set of Dirichlet series $f(s)=\sum_{n} a_{n} n^{-s}$ such that $\sigma_{u}(f) \leq 0$ coincides with the set of holomorphic functions on $\mathbb{C}_{0}$ that are bounded on $\mathbb{C}_{\varepsilon}$ for each $\varepsilon>0$ and that can be represented as a convergent Dirichlet series in $\mathbb{C}_{0}$. The second motivating result is the following improved Montel principle due to Bayart [4, Lemma 18] (see also Theorem 6.3.1 in [31]): If $\left(f_{k}\right)_{k}$ is a bounded sequence in $\mathcal{H}^{\infty}$, then there are $f \in \mathcal{H}^{\infty}$ and a subsequence $\left(f_{k(j)}\right)_{j}$ of the original sequence that converges uniformly to $f$ on $\mathbb{C}_{\varepsilon}$ for each $\varepsilon>0$. This result has important consequences for the topological structure of the space $\mathcal{H}_{+}^{\infty}$ of holomorphic functions on $\mathbb{C}_{0}$ that are bounded on $\mathbb{C}_{\varepsilon}$ for each $\varepsilon>0$ and that can be represented as a convergent Dirichlet series in $\mathbb{C}_{0}$, when $\mathcal{H}_{+}^{\infty}$ is endowed with its natural locally convex topology defined below. The aim of this paper is to investigate the structure

2010 Mathematics Subject Classification. Primary: 46A04, secondary: 30B50; 32A05; 46A03; 46A11; 46A $45 ; 47 \mathrm{~B} 33$

Key words and phrases. Dirichlet series; abscissas of convergence; Fréchet spaces; composition operators; topological algebras 
of the Fréchet space $\mathcal{H}_{+}^{\infty}$ both as a locally convex space and as a topological algebra as well as to study continuous composition operators on this space and composition operators that map a neighbourhood into a bounded subset.

The general theory of Dirichlet series was developed at the beginning of the last century by Bohr, Hardy, Landau and Riesz, among others. Recently the field showed remarkable advances, in particular combining functional analytical and complex analytical tools. We refer to the books [20] and [31], the articles [8], [13], [19] and [29], and the references therein for more information. If $\Omega$ is an open subset of $\mathbb{C}$, the space of holomorphic functions on $\Omega$ is denoted by $H(\Omega)$. Moreover, $H^{\infty}(\Omega)$ stands for the space of bounded holomorphic functions. Our notation for locally convex spaces, Banach spaces and functional analysis is standard. See e.g. [23] and [27]. We refer the reader to Mallios, [25], Michael [28] and Żelazko [33] for terminology concerning topological algebras. Necessary definitions will be recalled when needed later in the article.

A Dirichlet series is a series of the form $f(s)=\sum_{n} a_{n} n^{-s}$ with complex coefficients $a_{n}$ and variable $s \in \mathbb{C}$. The abscissas of convergence, uniform convergence and absolute convergence of $f$ are defined as follows (see [1], [22] and [31]):

$$
\begin{gathered}
\sigma_{c}(f):=\inf \left\{r \mid \sum_{n} a_{n} n^{-s} \text { converges on } \mathbb{C}_{r}\right\}, \\
\sigma_{u}(f):=\inf \left\{r \mid \sum_{n} a_{n} n^{-s} \text { converges uniformly on } \mathbb{C}_{r}\right\}, \\
\sigma_{a}(f):=\inf \left\{r \mid \sum_{n} a_{n} n^{-s} \text { converges absolutely on } \mathbb{C}_{r}\right\} .
\end{gathered}
$$

Here the infima are taken in the extended real line. When the Dirichlet series is nowhere convergent, the three abscissas are $+\infty$. We have $-\infty \leq \sigma_{c}(f) \leq \sigma_{u}(f) \leq \sigma_{a}(f) \leq \infty$. By the highly non-trivial result of Bohr [10] mentioned above $\sigma_{u}(f)$ coincides with the infimum of those $\sigma$ for which the function $f(s)=\sum_{n} a_{n} n^{-s}$, possibly by analytic continuation from a smaller half-plane, is analytic and bounded on $\mathbb{C}_{\sigma}$. Recall that $\mathcal{H}^{\infty}$ is the Banach space of all Dirichlet series $f(s)=\sum_{n} a_{n} n^{-s}$ that converges to a bounded analytic function on $\mathbb{C}_{0}$ endowed with the norm $\|f\|:=\sup _{s \in \mathbb{C}_{0}}|f(s)|$.

\section{The Fréchet Schwartz algebra $\mathcal{H}_{+}^{\infty}$}

We denote by $\mathcal{H}_{+}^{\infty}$ the space of all analytic functions on the half-plane $\mathbb{C}_{0}$ which are bounded on $\mathbb{C}_{\varepsilon}$ for each $\varepsilon>0$ and that can be represented as a convergent Dirichlet series $f(s)=\sum_{n} a_{n} n^{-s}$ in $\mathbb{C}_{0}$. It is endowed with the metrizable locally convex topology defined by the system of seminorms

$$
P_{\varepsilon}(f):=\sup _{s \in \mathbb{C}_{\varepsilon}}|f(s)|, \quad f \in \mathcal{H}_{+}^{\infty} .
$$

Endowed with this topology $\mathcal{H}_{+}^{\infty}$ is a Fréchet space, i.e. a complete metrizable locally convex space, that can be written as the projective limit $\mathcal{H}_{+}^{\infty}=\operatorname{proj}_{k} \mathcal{H}_{k}^{\infty}$, where $\mathcal{H}_{k}^{\infty}$ is the Banach space of all bounded analytic functions on $\mathbb{C}_{1 / k}$ that can be represented as a convergent Dirichlet series in $\mathbb{C}_{1 / k}$ endowed with the norm $\|g\|_{k}=\sup _{s \in \mathbb{C}_{1 / k}}|g(s)|, g \in \mathcal{H}_{k}^{\infty}$, and the linking maps $\pi_{k+1}^{k}: \mathcal{H}_{k+1}^{\infty} \rightarrow \mathcal{H}_{k}^{\infty}$ are restrictions. We denote by $\pi_{k}: \mathcal{H}_{+}^{\infty} \rightarrow \mathcal{H}_{k}^{\infty}$ the restriction map. We refer the reader to [23, Chapter 2] and [27, Chapter 24] for projective topologies 
and projective limits. As mentioned in the introduction, Bohr's fundamental theorem [10] (cf. [31, Theorem 6.2.3]) implies that the Banach space $\mathcal{H}^{\infty}$ of bounded Dirichlet series in $\mathbb{C}_{0}$ is contained in $\mathcal{H}_{+}^{\infty}$, and that the later space coincides with the space of all Dirichlet series $f(s)=\sum_{n} a_{n} n^{-s}$ such that $\sigma_{u}(f) \leq 0$. The space $\mathcal{H}_{+}^{\infty}$ is continuously included in the Fréchet space $H\left(\mathbb{C}_{0}\right)$.

Example 2.1 (1) The functions $f(s)=(s-1) /(s+1)$ and $f(a)=a^{-s}, a>1$ not a positive integer, belong to $H\left(\mathbb{C}_{0}\right)$, they are bounded in modulus by 1 , but they are not a convergent Dirichlet series, hence they do not belong to $\mathcal{H}_{+}^{\infty}$. See [31, page 140] and [30, page 297].

(2) The function $f(s)=\left(1-2^{-s}\right) \zeta(s)=\sum_{n}(-1)^{n-1} n^{-s-1}$ does not belong to $\mathcal{H}^{\infty}$, see [31, page 140]. However, $f \in \mathcal{H}_{+}^{\infty}$ since it is easy to see that $\sigma_{a}(f)=0$.

We recall that a Fréchet space $X$ with a basis of decreasing, absolutely convex 0-neighbourhoods $\left\{U_{n}\right\}_{n=1}^{\infty}$ is Schwartz if

$$
\forall n \in \mathbb{N} \exists m>n \forall \varepsilon>0 \exists F_{\varepsilon} \subset X \text { finite such that } U_{m} \subset F_{\varepsilon}+\varepsilon U_{n} .
$$

Therefore, a Fréchet space $X$ is Schwartz if and only if $X$ can be written as a projective limit via continuous linear linking operators $S_{n}: X_{n+1} \rightarrow X_{n}$, for $n \in \mathbb{N}$, with each $X_{n}$ a Banach space, such that, for every $n \in \mathbb{N}$, there exists $m>n$ with $\left(S_{m-1} \circ \ldots \circ S_{n}\right): X_{m} \rightarrow X_{n}$ a compact operator. Every nuclear Fréchet space is Schwartz, see [27, Corollary 28.5]. Further details about nuclear spaces and Schwartz spaces can be found in [23] and [27].

The definition of Köthe echelon spaces is needed in this section. A sequence $B=\left(b_{k}\right)_{k}$ of functions $b_{k}: I \rightarrow[0, \infty)$, with $I$ an infinite index set, is called a Köthe matrix on $I$ if $0 \leq b_{k}(i) \leq b_{k+1}(i)$, for all $i \in I$ and $k \in \mathbb{N}$, and if for each $i \in I$ there is $k \in \mathbb{N}$ such that $b_{k}(i)>0$. To each $p \in[1, \infty)$ we associate the linear space

$$
\lambda_{p}(B, I):=\left\{x \in \mathbb{C}^{I}: q_{k}^{(p)}(x):=\left(\sum_{i \in I}\left|b_{k}(i) x_{i}\right|^{p}\right)^{1 / p}<\infty, \quad \forall k \in \mathbb{N}\right\} .
$$

Elements $x \in \mathbb{C}^{I}$ are denoted by $x=\left(x_{i}\right)_{i}$. The spaces $\lambda_{p}(B, I)$, for $p \in[1, \infty[$, are called Köthe echelon spaces of order $p$; they are all Fréchet spaces (separable if $I$ is countable and reflexive if $p \neq 1$ ) relative to the increasing sequence of seminorms $q_{1}^{(p)} \leq q_{2}^{(p)} \leq \ldots$ In case $I=\mathbb{N}$, we simply write $\lambda_{p}(B)$. In this case $\lambda_{p}(B)$ has an unconditional basis. A space $\lambda_{p}(B)$ is Schwartz (resp. nuclear) if and only if for each $k \in \mathbb{N}$ there is $m>k$ such that $\left(a_{k}(i) / a_{m}(i)\right)_{i} \in c_{0}$ (resp. $\left.\left(a_{k}(i) / a_{m}(i)\right)_{i} \in \ell_{1}\right)$; see [27, Proposition 27.10 and Proposition 28.16]. Further details about Köthe echelon spaces and their duals can be found in [7] and [27, Chapter 27].

Theorem 2.2 The Fréchet space $\mathcal{H}_{+}^{\infty}$ is Schwartz, non-nuclear and the Dirichlet monomials $e_{n}(s)=n^{-s}, n \in \mathbb{N}$ are a Schauder basis of the space. Moreover, for each $m \in \mathbb{N}, \mathcal{H}_{+}^{\infty}$ contains an isomorphic copy of the space $H\left(\mathbb{D}^{m}\right)$ of holomorphic functions on the $m$-dimensional polydisc $\mathbb{D}^{m}$.

Proof. For each $k \in \mathbb{N}$ the restriction map $\pi_{k+1}^{k}: \mathcal{H}_{k+1}^{\infty} \rightarrow \mathcal{H}_{k}^{\infty}$ is compact by an application of the improved Montel principle of Bayart [4, Lemma 18] (see also Theorem 6.3.1 in [31]): If $\left(f_{j}\right)_{j}$ is a bounded sequence in $\mathcal{H}_{k+1}^{\infty}$, then there are $f \in \mathcal{H}_{k+1}^{\infty}$ and a subsequence $\left(f_{j(s)}\right)_{s}$ of the original sequence that converges uniformly to $f$ in $\mathcal{H}_{k}^{\infty}$. Therefore the space $\mathcal{H}_{+}^{\infty}=\operatorname{proj}_{k} \mathcal{H}_{k}^{\infty}$ is Fréchet Schwartz. 
To prove that $\mathcal{H}_{+}^{\infty}$ is not nuclear, we show that it contains a complemented subspace isomorphic to a non-nuclear Köthe echelon space of order one. Let $\mathcal{P}$ be the set of prime numbers $p_{1}<p_{2}<p_{n}<\ldots$. Consider the Köthe matrix $B=\left(b_{k}\right)_{k}$ on $\mathcal{P}$ by $b_{k}(p):=$ $p^{-1 / k}, p \in \mathcal{P}, k \in \mathbb{N}$. Define $R: \mathcal{H}_{+}^{\infty} \rightarrow \lambda_{1}(B, \mathcal{P})$ by $f(s)=\sum_{n} a_{n} n^{-s} \rightarrow R(f):=\left(a_{p}\right)_{p \in \mathcal{P}}$ and $S: \lambda_{1}(B, \mathcal{P}) \rightarrow \mathcal{H}_{+}^{\infty}$ by $S\left(\left(a_{p}\right)_{p \in \mathcal{P}}\right):=\sum_{p \in \mathcal{P}} a_{p} p^{-s}$. The operator $R$ is continuous by Bohr's inequality [31, Theorem 6.2.4]. Indeed, fix $k \in \mathbb{N}$. If $f=\sum_{n} a_{n} n^{-s} \in \mathcal{H}_{+}^{\infty}$, then $f_{k}:=\sum_{n}\left(a_{n} n^{-1 / k}\right) n^{-s} \in \mathcal{H}^{\infty}$, hence $\sum_{p \in \mathcal{P}}\left|a_{p}\right| p^{-1 / k} \leq \sup _{s \in \mathbb{C}_{0}}\left|f_{k}(s)\right|=\sup _{s \in \mathbb{C}_{1 / k}}|f(s)|=$

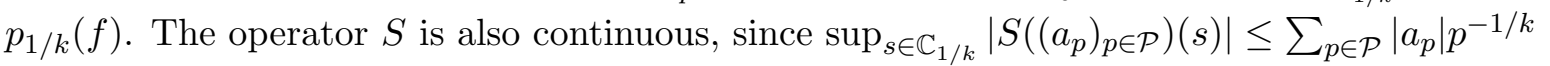
for each $\left(a_{p}\right)_{p \in \mathcal{P}} \in \lambda_{1}(B, \mathcal{P})$. Now $R \circ S$ coincides with the identity on $\lambda_{1}(B, \mathcal{P})$. This implies that that $S$ is an isomorphism into and that $S \circ R$ is a projection in $\mathcal{H}_{+}^{\infty}$ with range $S\left(\lambda_{1}(B, \mathcal{P})\right)$.

The Köthe echelon space $\lambda_{1}(B, \mathcal{P})$ is not nuclear. If it were, by the Grothendieck Pietsch criterion [27, Proposition 28.16], there would be $k>1$ such that $\sum_{p \in \mathcal{P}}\left(b_{1}(p) / b_{k}(p)\right)<\infty$, but $b_{1}(p) / b_{k}(p)=1 / p^{1-1 / k}>1 / p$ and $\sum_{p \in \mathcal{P}} \frac{1}{p}=\infty$.

For each $f(s)=\sum_{n} a_{n} n^{-s} \in \mathcal{H}_{+}^{\infty}$, we have $f_{1}(s):=\sum_{n}\left(a_{n} / n\right) n^{-s} \in \mathcal{H}^{\infty}$. We can apply [31, Theorem 6.1.1] to conclude that $\left|a_{n}\right| \leq n \sup _{s \in \mathbb{C}_{1}}|f(s)|$ for each $n \in \mathbb{N}$, and hence all coefficient functionals $u_{n}: \mathcal{H}_{+}^{\infty} \rightarrow \mathbb{C}, f \rightarrow a_{n}$ of the Dirichlet monomials $e_{n}$ are continuous. Moreover, $\sigma_{u}(f) \leq 0$ for each $f(s)=\sum_{n} a_{n} n^{-s} \in \mathcal{H}_{+}^{\infty}$. This implies that the partial sums $S_{N}(f)(s)=\sum_{n=1}^{N} a_{n} n^{-s}$ converge to $f(s)$ uniformly on $\mathbb{C}_{\varepsilon}$ for each $\varepsilon>0$. This means that the sequence $\left(S_{N}(f)\right)_{N}$ converges to $f$ in $\mathcal{H}_{+}^{\infty}$; in other words that $f$ can be written as a series $\sum_{n} a_{n} e_{n}$ converging in $\mathcal{H}_{+}^{\infty}$. Thus $\left(e_{n}\right)_{n}$ is a Schauder basis of the space.

Fix $m \in \mathbb{N}$. Let $2=p_{1}<\ldots<p_{m}$ be the first $m$ prime numbers. The map $\Psi$ : $\mathbb{C}_{0} \rightarrow \mathbb{D}^{m}, \Psi(s):=\left(2^{-s}, 3^{-s}, \ldots,\left(p_{m}\right)^{-s}\right)$ satisfies that $\Psi\left(\mathbb{C}_{\varepsilon}\right)$ is dense in $\left\{z=\left(z_{1}, \ldots, z_{m}\right) \in\right.$ $\left.\mathbb{C}^{m}|| z_{i} \mid<\left(p_{i}\right)^{-\varepsilon}, z_{i} \neq 0, i=1, \ldots m\right\}$. This implies that the map $\Delta: H\left(\mathbb{D}^{m}\right) \rightarrow \mathcal{H}_{+}^{\infty}$ given by $\Delta(g)(s):=g\left(2^{-s}, 3^{-s}, \ldots,\left(p_{m}\right)^{-s}\right)$ defines a topological isomorphism into its image.

Proposition 2.3 The operator $T$ defined by $f(s)=\sum_{n} x_{n} n^{-s} \rightarrow\left(x_{n}\right)_{n}$ defines a continuous non-surjective injection from $\mathcal{H}_{+}^{\infty}$ into $\lambda_{2}(D)$ for the Köthe matrix $D=\left(d_{k}\right), d_{k}(n)=$ $1 / n^{1 / k}, k, n \in \mathbb{N}$. Moreover, $\mathcal{H}_{+}^{\infty}$ is not isomorphic to $\lambda_{2}(D)$.

Proof. For each $k \in \mathbb{N}$ and $f(s)=\sum_{n} x_{n} n^{-s} \in \mathcal{H}_{+}^{\infty}$, the function $f_{k}(s)=\sum_{n}\left(x_{n} n^{-(1 / k)}\right) n^{-s}$ belongs to $\mathcal{H}^{\infty}$. We can apply Carlson's identity [31, Theorem 6.2.5] to get $\sum_{n}\left|x_{n} n^{-(1 / k)}\right|^{2} \leq$ $\sup _{s \in \mathbb{C}_{0}}\left|f_{k}(s)\right|=\sup _{s \in \mathbb{C}_{1 / k}}\left|f_{k}(s)\right|$. This proves the operator $T$ is well defined and continuous. It is clearly injective. The operator $T$ is not surjective because $\left(1 / n^{1 / 2}\right)_{n} \in \lambda_{2}(D)$, but $\sum_{n} \frac{1}{n^{1 / 2}} n^{-s} \notin \mathcal{H}_{+}^{\infty}$.

Suppose now that $\mathcal{H}_{+}^{\infty}$ is isomorphic to $\lambda_{2}(D)$ for the matrix $D$ as defined in the statement. This implies that the space $\mathcal{H}_{+}^{\infty}$ is hilbertisable, in the sense that it admits a fundamental system of seminorms defined by scalar products. Therefore, the complemented subspace $\lambda_{1}(B, \mathcal{P})$ constructed in the proof of Theorem 2.2 is also hilbertisable. However, a Köthe echelon space $\lambda_{1}(A)$ of order one is hilbertisable if and only if it is nuclear. Here is an argument to see this statement: if $\lambda_{1}(A)$ is hilbertisable, then for each $k$ there is $l>k$ such that the linking map $\ell_{1}\left(a_{l}\right) \rightarrow \ell_{1}\left(a_{k}\right)$ factors through a Hilbert space, see e.g. [21, Proposition 1.4]. Every bounded operator from $\ell_{1}$ into a Hilbert space is absolutely summing as a consequence of Grothendieck's inequality; see e.g. [14, p. 181]. Consequently, for each $k$ there is $l>k$ such that the linking map $\ell_{1}\left(a_{l}\right) \rightarrow \ell_{1}\left(a_{k}\right)$ is absolutely summing, and the space $\lambda_{1}(A)$ is nuclear. 
Remark 2.4 Hölder's inequality implies that the Köthe echelon space $\lambda_{2}(D)$ of order two for the Köthe matrix $D=\left(d_{k}\right), d_{k}(n)=1 / n^{1 / k}, k, n \in \mathbb{N}$, that appears in Proposition 2.3 is continuously contained in the Köthe echelon space $\lambda_{1}(C)$ of order one for the Köthe matrix $C=\left(c_{k}\right), c_{k}(n)=1 / n^{1 / 2+1 / k}, k, n \in \mathbb{N}$. Compare with [26, Theorem 2.2].

Lemma 2.5 For each $\delta>0$ and each $k \in \mathbb{N}$ there is a Dirichlet polynomial $h$ such that $h(\delta / 2)=0$ and $\sup _{s \in \mathbb{C}_{\delta}}|1-h(s)|<1 / k$.

Proof. Fix $\delta<0$. We apply [2, Theorem 3.1] to $K=\{0\} \subset\{s \in \mathbb{C} \mid \operatorname{Re} s \leq 0\}, f(s)=1$ for each $s \in \mathbb{C}, g(s)=0$ on $K, \sigma=\delta / 2$ and $\varepsilon=1 /(2 k)$ to find a Dirichlet polynomial $g$ such that $|g(0)|<1 /(2 k), g(s)=\sum_{n=1}^{N} a_{n} n^{-s}$ and

$$
\left|1-a_{1}\right|+\sum_{n=1}^{N}\left|a_{n}\right| n^{-\delta / 2}<1 /(2 k) .
$$

Observe that this implies $|1-g(s)| \leq 1 /(2 k)$ for each $s \in \mathbb{C}_{\delta / 2}$. Set $\tilde{g}(s):=g(s)-g(0)$. For $s \in \mathbb{C}_{\delta / 2}$ we get $|1-\tilde{g}(s)| \leq|1-g(s)|+|g(0)|<1 / k$. Now $h(s):=g(s-(\delta / 2))$ is a Dirichlet polynomial that satisfies $h(\delta / 2)=\tilde{g}(0)=0$. Moreover, if $s \in \mathbb{C}_{\delta}$, then $s-(\delta / 2) \in \mathbb{C}_{\delta / 2}$ and $|1-h(s)|=|1-g(s-(\delta / 2))|<1 / k$.

A locally convex algebra is called a $Q$-algebra if the sets of invertible elements is open.

Theorem 2.6 (1) The Fréchet space $\mathcal{H}_{+}^{\infty}$ is a multiplicatively convex Fréchet algebra for the pointwise product.

(2) An element $f \in \mathcal{H}_{+}^{\infty}$ is invertible if and only if $\inf _{s \in \mathbb{C}_{\varepsilon}}|f(s)|>0$ for each $\varepsilon>0$.

(3) The space $\mathcal{H}_{+}^{\infty}$ is not a $Q$-algebra.

Proof. (1) It is clear that $\mathcal{H}_{+}^{\infty}$ is a Fréchet algebra since it is the countable intersection of Banach algebras by [31, Theorem 6.2.1]. Moreover it is multiplicatively convex as the seminorms defining the topology satisfy $P_{\varepsilon}(f g) \leq P_{\varepsilon}(f) P_{\varepsilon}(g)$ for each $\varepsilon>0$ and each $f, g \in$ $\mathcal{H}_{+}^{\infty}$.

(2) If $f \in \mathcal{H}_{+}^{\infty}$ is invertible, there is $g \in \mathcal{H}_{+}^{\infty}$ such that $f(s) g(s)=1$ for each $s \in \mathbb{C}_{0}$. For each $\varepsilon>0$ and each $s \in \mathbb{C}_{\varepsilon}$, we have $|f(s)|=1 /|g(s)| \geq 1 / \sup _{s \in \mathbb{C}_{\varepsilon}}|g(s)|$ and $\inf _{s \in \mathbb{C}_{\varepsilon}}|f(s)|>$ 0 . Conversely, suppose that $f \in \mathcal{H}_{+}^{\infty}$ satisfies $\inf _{s \in \mathbb{C}_{\varepsilon}}|f(s)|>0$ for each $\varepsilon>0$. We have $g:=1 / f \in H\left(\mathbb{C}_{0}\right)$. Moreover, for each $\varepsilon>0$ and $s \in \mathbb{C}_{\varepsilon}$, we have $g(s)=g_{\varepsilon}(s-\varepsilon)$ and $g$ is bounded in $\mathbb{C}_{\varepsilon}$. Finally, for $s \in \mathbb{C}_{1}$, we have $g(s)=g_{1}(s-1)$, which implies that $g$ is a convergent Dirichlet series for Re $s$ large enough. We have shown $g \in \mathcal{H}_{+}^{\infty}$ and $f g=1$.

(3) Proceeding by contradiction, assume that $\mathcal{H}_{+}^{\infty}$ is a $Q$-algebra. Then 1 is an interior point of the set of invertible elements in the algebra $\mathcal{H}_{+}^{\infty}$. There are $\delta>0$ and $k \in \mathbb{N}$ such that every $h \in \mathcal{H}_{+}^{\infty}$ such that $\sup _{s \in \mathbb{C}_{\delta}}|1-h(s)|<1 / k$ is invertible in $\mathcal{H}_{+}^{\infty}$, in particular $h(s) \neq 0$ for each $s \in \mathbb{C}_{0}$. This fact contradicts the statement proved in Lemma 2.5.

Example 2.7 Clearly every invertible element of $\mathcal{H}^{\infty}$ is also invertible in $\mathcal{H}_{+}^{\infty}$. The Dirichlet polynomial $f(s)=1-2^{-s}$ belongs to $\mathcal{H}^{\infty}$, it is invertible in $\mathcal{H}_{+}^{\infty}$ by Theorem 2.6 , but not in $\mathcal{H}^{\infty}$ by [31, Theorem 6.2.1], since $\inf _{s \in \mathbb{C}_{0}}|f(s)|=0$. The inverse of $f(s)$ is $1+2^{-s}+4^{-s}+6^{-s}+\ldots$ An example of an absolutely convergent Dirichlet series $g(s)$ without zeros on $\overline{\mathbb{C}_{0}}:=\{s \in$ $\mathbb{C} \mid \operatorname{Re} s \geq 0\}$ satisfying inf $s \in \overline{\mathbb{C}_{0}}|g(s)|=0$ can be seen in [16, p. 1074]. The Dirichlet monomial $g(s)=2^{-s}$ is an invertible element in the space $H\left(\mathbb{C}_{0}\right)$, but it is not invertible in $\mathcal{H}_{+}^{\infty}$, for example by [6, Proposition 1.1]. 
Bohr made in [9] a following fundamental contribution, that is called nowadays the Bohr transform $\mathcal{B}$. To describe it, we denote $\mathbb{N}_{0}:=\mathbb{N} \cup\{0\}$, and by $\mathbb{N}_{0}^{(\mathbb{N})}$ the set of all multi-indices $\alpha=\left(\alpha_{1}, \ldots, \alpha_{n}, 0,0,0, \ldots\right)$ with $\alpha_{i} \in \mathbb{N}_{0}$ for each $i$. If $p=\left(p_{k}\right)_{k}$ is the sequence of prime numbers and $\alpha \in \mathbb{N}_{0}^{(\mathbb{N})}$, then we write $p^{\alpha}:=p_{1}^{\alpha_{1}} \ldots p_{n}^{\alpha_{n}}$. The Bohr transform associates to each Dirichlet series $f(s)=\sum_{n} x_{n} n^{-s}$ a formal power series $\mathcal{B}(f):=\sum_{\alpha \in \mathbb{N}_{0}^{(\mathbb{N})}} c_{\alpha} z^{\alpha}$ by setting $a_{n}=c_{\alpha}$ if $n=p^{\alpha}$. This bijection preserves topological and measure properties. For example this transform establishes a Banach algebra isometry between the space $\mathcal{H}^{\infty}$ and the Banach space $H^{\infty}\left(B_{c_{0}}\right)$ of bounded holomorphic functions on the unit ball $B_{c_{0}}$ of $c_{0}$. See [4, Section 2], [11], [12], [31, Chapter 4]. We refer to [15] for infinite dimensional holomorphy. The following result is a direct consequence of Lemma 2.2, Proposition 2.3 and formula (1.4) in [11].

Proposition 2.8 The Bohr transform $\mathcal{B}$ maps $f(s)=\sum_{n} x_{n} n^{-s} \in \mathcal{H}_{+}^{\infty}$ surjectively into a formal power series such that for each $\varepsilon>0$ there is a function $g_{\varepsilon}$ in the space $H^{\infty}\left(B_{\ell_{\infty}}\right)$ of bounded holomorphic functions on the unit ball $B_{\ell_{\infty}}$ of $\ell_{\infty}$ such that the coefficients of the associated formal Taylor series of $g_{\varepsilon}$ are $\left(a_{p^{\alpha}} / p^{\varepsilon \alpha}\right)_{\alpha \in \mathbb{N}_{0}^{N}}$.

\section{Composition operators on the space $\mathcal{H}_{+}^{\infty}$}

Composition operators on the Hilbert space $\mathcal{H}^{2}$ of Dirichlet series were characterized by Gordon and Hedenmalm [18]; see also Section 3 of Queffélec's survey [30]. On the other hand, Bayart [4, after Corollary 2, p. 217] and [3, p. 65] proved that an analytic map $\phi: \mathbb{C}_{0} \rightarrow \mathbb{C}_{0}$ defines a continuous composition operator $C_{\phi}(f):=f \circ \phi$ on the Banach space $\mathcal{H}^{\infty}$ if and only if $\phi(s)=c_{0} s+\sum_{n} c_{n} n^{-s}$ with $c_{0}$ a non-negative integer and $\varphi(s):=\sum_{n} c_{n} n^{-s}$ a Dirichlet series convergent in some half-plane. Bayart also proved in [4, Theorem 18] that $C_{\phi}$ is compact on $\mathcal{H}^{\infty}$ if and only if $\phi\left(\mathbb{C}_{0}\right) \subset \mathbb{C}_{\varepsilon}$ for some $\varepsilon>0$. The study of composition operators on $\mathcal{H}^{\infty}$ continued in [24] and [32].

Recall that an operator $T: E \rightarrow E$ on a locally convex space $E$ is called bounded if there is a 0-neighbourhood $U$ in $E$ such that $T(U)$ is bounded in $E$. Every bounded operator is continuous. In this section we characterize continuous and bounded composition operators $C_{\phi}$ on the Fréchet space $\mathcal{H}_{+}^{\infty}$.

Lemma 3.1 (1) Let $\phi: \mathbb{C}_{0} \rightarrow \mathbb{C}_{0}$ be analytic. If $C_{\phi}(f)=f \circ \phi$ is a Dirichlet series convergent in some half-plane for each Dirichlet polynomial $f$, then $\phi(s)=c_{0} s+\sum_{n} c_{n} n^{-s}$ with $c_{0} a$ non-negative integer and $\varphi(s):=\sum_{n} c_{n} n^{-s}$ a Dirichlet series convergent in some half-plane.

(2) Let $\phi: \mathbb{C}_{0} \rightarrow \mathbb{C}_{0}$ be an analytic function such that $\phi(s)=c_{0} s+\sum_{n} c_{n} n^{-s}$ with $c_{0} a$ non-negative integer and $\varphi(s):=\sum_{n} c_{n} n^{-s}$ a Dirichlet series convergent in some half-plane. If $f(s)=\sum_{n} a_{n} n^{-s}$ is a Dirichlet series such that $\sigma_{u}(f) \leq 0$, then $f \circ \phi$ is a Dirichlet series convergent in some half-plane.

Proof. Part (1) follows from the proof of the necessity in Theorem A, pages 315-317, in [18]. The proof of part (2) is obtained modifying the proof of the sufficiency in Theorem A, pages 318-319, in [18]. Only the case $c_{0}=0$ requires some inspection. If $\varphi(s)$ is constant, the conclusion is easy. If $\varphi(s)$ is not constant, [17, Lemma 2] implies $\operatorname{Re} c_{1}>0$, the series $\sum_{n} a_{n} n^{-s}$ converges uniformly in $\mathbb{C}_{\operatorname{Re} c_{1} / 2}$ and the series formally obtained expanding $\sum_{n} a_{n} n^{-\phi(s)}$ converges in $\mathbb{C}_{\eta}$ for $\eta>0$ sufficiently large. A similar argument is implicit in 
Bayart's result [4, after Corollary 2, p. 217] that if $\phi$ satisfies the assumptions in (2), then $C_{\phi}(g)$ is a convergent Dirichlet function for each $f \in \mathcal{H}^{\infty}$.

The next Lemma is a particular case of [18, Theorem 4.2] (see also [4, Lemma 12] and [17, Lemma 2]).

Lemma 3.2 Let $\phi: \mathbb{C}_{0} \rightarrow \mathbb{C}_{0}$ be an analytic function such that $\phi(s)=c_{0} s+\sum_{n} c_{n} n^{-s}$ with $c_{0}$ a non-negative integer and $\varphi(s):=\sum_{n} c_{n} n^{-s}$ a Dirichlet series convergent in some half-plane. Then

(1) If $\varphi(s)=c_{1}$ for each $s \in \mathbb{C}_{0}$, then $\operatorname{Re} c_{1} \geq 0$.

(2) If $\varphi(s)$ is not constant, then it can be extended to an analytic function $\varphi: \mathbb{C}_{0} \rightarrow \mathbb{C}_{0}$. such that for each $\theta>0$ there is $\eta>0$ such that $\varphi\left(\mathbb{C}_{\theta}\right) \subset \mathbb{C}_{\eta}$.

(3) If $\varphi(s)$ is not constant, then $\operatorname{Re} c_{1}>0$.

Lemma 3.3 Let $\phi: \mathbb{C}_{0} \rightarrow \mathbb{C}_{0}$ be an analytic function such that $\phi(s)=c_{0} s+\sum_{n} c_{n} n^{-s}$ with $c_{0}$ a non-negative integer and $\varphi(s):=\sum_{n} c_{n} n^{-s}$ a Dirichlet series convergent in some half-plane. Then for each $\varepsilon>0$ there is $\delta>0$ such that $\phi\left(\mathbb{C}_{\varepsilon}\right) \subset \mathbb{C}_{\delta}$.

Proof. Suppose first that $\varphi$ is not constant. By Lemma $3.2(2)$, given $\varepsilon>0$ there is $\eta>0$ such that $\varphi\left(\mathbb{C}_{\varepsilon}\right) \subset \mathbb{C}_{\eta}$. This implies $\phi\left(\mathbb{C}_{\varepsilon}\right) \subset \mathbb{C}_{c_{0} \varepsilon+\eta}$.

Now assume that $\varphi(s)=c_{1}$ for all $s \in \mathbb{C}_{0}$. In case $\operatorname{Re} c_{1}>0$, we get $\phi\left(\mathbb{C}_{0}\right) \subset \mathbb{C}_{\operatorname{Re} c_{1}}$. On the other hand, if $c_{1}=i \tau, \tau \in \mathbb{R}$, we must have $c_{0}>0$, since otherwise $\phi\left(\mathbb{C}_{0}\right) \subset \mathbb{C}_{0}$ would not be satisfied. Accordingly $\phi\left(\mathbb{C}_{\varepsilon}\right) \subset \mathbb{C}_{c_{0} \varepsilon}$ for each $\varepsilon>0$.

Proposition 3.4 Let $\phi: \mathbb{C}_{0} \rightarrow \mathbb{C}_{0}$ be analytic.

(1) The composition operator $C_{\phi}$ is continuous on the space $\mathcal{H}_{+}^{\infty}$ if and only if $\phi(s)=$ $c_{0} s+\sum_{n} c_{n} n^{-s}$ with $c_{0}$ a non-negative integer and $\varphi(s):=\sum_{n} c_{n} n^{-s}$ a Dirichlet series convergent in some half-plane.

(2) Suppose that the composition operator $C_{\phi}$ is continuous on the space $\mathcal{H}_{+}^{\infty}$. Then $C_{\phi}$ is bounded on $\mathcal{H}_{+}^{\infty}$ if and only if there is $\varepsilon>0$ such that $\phi\left(\mathbb{C}_{0}\right) \subset \mathbb{C}_{\varepsilon}$.

Proof. (1) If $\phi(s)=c_{0} s+\sum_{n} c_{n} n^{-s}$ with $c_{0}$ a non-negative integer and $\varphi(s):=\sum_{n} c_{n} n^{-s}$ is a Dirichlet series convergent in some half-plane, then for each $\varepsilon>0$ there is $\delta>0$ such that $\phi\left(\mathbb{C}_{\varepsilon}\right) \subset \mathbb{C}_{\delta}$ by Lemma 3.3. Therefore for each $\varepsilon>0$ there is $\delta>0$ such that, for each $f \in \mathcal{H}_{+}^{\infty}$, the function $C_{\phi}(f)$ is a Dirichlet series convergent in some half-plane by Lemma 3.1 $(2)$, and moreover $\sup _{s \in \mathbb{C}_{\varepsilon}}\left|C_{\phi}(f)(s)\right| \leq \sup _{s \in \mathbb{C}_{\delta}}|f(s)|$. This implies $C_{\phi}(f) \in \mathcal{H}_{+}^{\infty}$ and that $C_{\phi}: \mathcal{H}_{+}^{\infty} \rightarrow \mathcal{H}_{+}^{\infty}$ is continuous.

Conversely, if $C_{\phi}$ is continuous on the space $\mathcal{H}_{+}^{\infty}$, then $C_{\phi}(f)$ is a Dirichlet series convergent in some half-plane for each Dirichlet polynomial $f$. The conclusion follows from Lemma 3.1 (1).

(2) If there is $\varepsilon>0$ such that $\phi\left(\mathbb{C}_{0}\right) \subset \mathbb{C}_{\varepsilon}$, then $\sup _{s \in \mathbb{C}_{0}}\left|C_{\phi}(f)(s)\right| \leq \sup _{s \in \mathbb{C}_{\varepsilon}}|f(s)|$ for each $f \in \mathcal{H}_{+}^{\infty}$. This implies that $C_{\phi}(U)$ is bounded in $\mathcal{H}_{+}^{\infty}$ for the neighbourhood $U:=\{f \in$ $\left.\mathcal{H}_{+}^{\infty}\left|\sup _{s \in \mathbb{C}_{\varepsilon}}\right| f(s) \mid \leq 1\right\}$.

Conversely suppose that the operator $C_{\phi}$ is bounded on $\mathcal{H}_{+}^{\infty}$ for an analytic map $\phi: \mathbb{C}_{0} \rightarrow$ $\mathbb{C}_{0}$ such that $\phi(s)=c_{0} s+\sum_{n} c_{n} n^{-s}$ with $c_{0}$ a non-negative integer and $\varphi(s):=\sum_{n} c_{n} n^{-s}$ a 
Dirichlet series convergent in some half-plane. Since $C_{\phi}$ is bounded, there is $\varepsilon>0$ such that for each $\delta>0$ there is $M_{\delta}>0$ such that

$$
\sup _{s \in \mathbb{C}_{\delta}}|f(\phi(s))| \leq M_{\delta} \sup _{s \in \mathbb{C}_{\varepsilon}}|f(s)| \quad \text { for each } \quad f \in \mathcal{H}_{+}^{\infty} .
$$

Evaluating this condition for each Dirichlet monomial $n^{-s}$, we get that for each $\delta>0$ and each $n \in \mathbb{N}, \sup _{s \in \mathbb{C} \delta} n^{-\operatorname{Re} \phi(s)} \leq M_{\delta} n^{-\varepsilon}$. Hence, for each $\delta>0$ and each $s \in \mathbb{C}_{\delta}$, we have $n^{\operatorname{Re} \phi(s)} \geq\left(1 / M_{\delta}\right) n^{\varepsilon}$ for each $n \in \mathbb{N}$. Therefore $\operatorname{Re} \phi(s) \geq \varepsilon$ for each $s \in \mathbb{C}_{\delta}$. Since $\delta>0$ is arbitrary, we conclude $\phi\left(\mathbb{C}_{0}\right) \subset \mathbb{C}_{\varepsilon}$.

Proposition 3.4 and the results of Bayart [4] mentioned above imply that the composition operator $C_{\phi}$ is continuous (resp. bounded) on the space $\mathcal{H}_{+}^{\infty}$ if and only it is continuous (resp. compact) on the Banach space $\mathcal{H}^{\infty}$.

Example 3.5 (i) If $\phi(s)=c_{0} s+i \tau, s \in \mathbb{C}_{0}$, with $c_{0}$ a positive integer and $\tau \in \mathbb{R}$, then the operator $C_{\phi}$ is continuous but not bounded in $\mathcal{H}_{+}^{\infty}$.

(ii) If $\phi(s)=c_{0} s+c_{1}+c_{2} 2^{-s}$, with $c_{0}$ a positive integer and $c_{2} \neq 0$, then $C_{\phi}$ is bounded in $\mathcal{H}_{+}^{\infty}$ if and only if $\operatorname{Re} c_{1}>\left|c_{2}\right|$. This is a consequence of a more general result due to Bayart [5, Corollary 2].

Acknowledgement. The research of this paper was partially supported by the projects MTM2013-43540-P and MTM2016-76647-P. This paper was written during the author's stay at the Katholische Universität Eichstätt-Ingolstadt (Germany). The support of the Alexander von Humboldt Foundation is greatly appreciated.

\section{References}

[1] T.M. Apostol: Introduction to analytic number theory. Springer-Verlag, New YorkHeidelberg, 1976.

[2] R. Aron, F. Bayart, P.M. Gauthier, M. Maestre, V. Nestoridis: Dirichlet approximation and universal Dirichlet series, arXiv:1608.06457v1. 2016.

[3] F. Bayart: Opérateurs de composition sur les espaces de séries de Dirichlet et problèmes d'hypercyclicité simultanée, Thése de l'Université Lille 1, 2002.

[4] F. Bayart: Hardy spaces of Dirichlet series and their composition operators, Monatsh. Math. 136 (2002), 203-236.

[5] F. Bayart: Compact composition operators on a Hilbert space of Dirichlet series, Illinois J. Math. 47 (2003), 725-743.

[6] F. Bayart, A. Mouze: Division et composition dans l'anneau des séries de Dirichlet analytiques, Ann. Inst. Fourier (Grenoble) 53 (2003), 2039-2060.

[7] K.D. Bierstedt, R.G. Meise, W.H. Summers, Köthe sets and Köthe sequence spaces, In: "Functional Analysis, Holomorphy and Approximation Theory" (Rio de Janeiro, 1980), North Holland Math. Studies 71 (1982), pp. 27-91. 
[8] H.P. Boas: The football player and the infinite series, Notices of the Amer. Math. Soc. 44 (1997) 1430-1435.

[9] H. Bohr: Über die Bedeutung der Potenzreihen unendlich vieler Variabeln in der Theorie der Dirichletschen Reihen $\sum \frac{a_{n}}{n^{s}}$, Nachr. Ges. Wiss. Göttingen, Math. Phys. Kl. 441-488 (1913).

[10] H. Bohr: Über die gleichmige Konvergenz Dirichletscher Reihen, J. Reine Angew. Math. 143 (1913), 203211.

[11] A. Defant, D. García, M. Maestre: New strips of convergence for Dirichlet series, Publ. Mat. 54 (2010), 369-388.

[12] A. Defant, D. García, M. Maestre, D. Pérez-García: Bohr's strip for vector valued Dirichlet series, Math. Ann. 342 (2008), no. 3, 533-555.

[13] A. Defant, D. García, M. Maestre, P. Sevilla-Peris: Bohr's strips for Dirichlet series in Banach spaces, Funct. Approx. Comment. Math. 44 (2011), 165-189.

[14] J. Diestel, Sequences and Series in Banach Spaces, Springer, New York, 1984.

[15] S. Dineen, Complex Analysis on infinite-dimensional spaces, Springer, London, 1999.

[16] D.A. Edwards, On absolutely convergent Dirichlet series, Proc. Amer. Math. Soc. 8 (1957), 1067-1074.

[17] C. Finet, H. Queffélec: Numerical range of composition operators on a Hilbert space of Dirichlet series, Linear Algebra Appl. 377 (2004), 1-10.

[18] J. Gordon, H. Hedenmalm: The composition operators on the space of Dirichlet series with square summable coefficients, Michigan Math. J. 46 (1999), 313-329.

[19] H. Hedenmalm: Dirichlet series and functional analysis, pp. 673-684 in The legacy of Niels Henrik Abel, Springer, Berlin, 2004.

[20] H. Helson: Dirichlet series. Henry Helson, Berkeley, CA, 2005.

[21] R. Hollstein, Generalized Hilbert spaces, Results in Math. 8 (1985), 95-115.

[22] G.J.O. Jameson: The prime number theorem, Cambridge University Press, Cambridge, 2003.

[23] H. Jarchow, Locally Convex Spaces, B.G. Teubner, Stuttgart, 1981.

[24] P. Lefévre: Essential norms of weighted composition operators on the space $\mathcal{H}^{\infty}$ of Dirichlet series, Studia Math. 191 (2009), 57-66.

[25] A. Mallios: Topological Algebras. Selected Topics, North-Holland Math. studies 124, North-Holland, 1986.

[26] B. Maurizi, H. Queffélec: Some remarks on the algebra of bounded Dirichlet series, J. Fourier Anal. Appl. 16 (2010), 676-692. 
[27] R. Meise and D. Vogt.: Introduction to Functional Analysis, The Clarendon Press Oxford University Press, New York, 1997.

[28] E. Michael: Localy multiplicatively-convex topological algebras, Memoirs Amer. Math. Soc. 11, 1952.

[29] H. Queffélec: Bohr's vision of ordinary Dirichlet series; old and new results. J. Anal. 3 (1995), 43-60.

[30] H. Queffélec: Espaces de séries de Dirichlet et leurs opérateurs de composition, Ann. Math. Blaise Pascal 22 (2015), 267-344.

[31] H. Queffélec, M. Queffélec: Diophantine Approximation and Dirichlet series, Hindustain Book Agency, New Delhi, 2013.

[32] M. Wang, X. Yao: Topological structure of the space of composition operators on $\mathcal{H}^{\infty}$ of Dirichlet series, Arch. Math. (Basel) 106 (2016), 471-483.

[33] W. Żelazko: Selected topics in topological algebras. Lectures 1969/1970. Lecture Notes Series, No. 31. Matematisk Institut, Aarhus Universitet, Aarhus, 1971. iii+176 pp.

\section{Author's address:}

Instituto Universitario de Matemática Pura y Aplicada IUMPA, Universitat Politècnica de València, E-46071 Valencia, SPAIN

email:jbonet@mat.upv.es 